\title{
Stochastic Damage Constitutive Law of Concrete under Uniaxial Compression
}

\author{
XIE Ming ${ }^{1, a}$, HAO Huiying ${ }^{1, b}$ \\ ${ }^{1}$ Shaanxi Key Laboratory of Safety and Durability of Concrete Structures, Xijing University, \\ Xi'an, 710123, P.R. China \\ a kpxtx@163.com \\ bfractal_x@163.com
}

Keywords: uniaxial compression, stochastic damage, microscopic model, failure mechanism Abstract: Based on the uniaxial tensile mesoscopic spring model of concrete, a uniaxial compression microscopic spring model is introduced. Considering the effect of material composition and horizontal deformation of concrete, a stochastic constitutive damage model of concrete is built. The effect of stiffness ratio on damage index is researched and the mechanism of fracture is studied. Based on the research of different failure modes of concrete with different strength grade, the stochastic damage constitutive equations of super high strength and high performance concrete, high strength concrete and normal concrete are built respectively to establish the unified stochastic damage constitutive model. Comparing with existing damage constitutive law and experiment results preliminarily, the feasibility of fractal damage constitutive law is verified.

\section{Introduction}

Concrete is a kind of complex composite materials, the material physical properties and its components exist difference, which is on the macroscopic mechanical properties of concrete is one of the reasons for presence of discreteness and randomness. This article is based on random constitutive model for concrete under uniaxial tension ${ }^{[1]}$, the advantage of the characteristics of the lateral deformation of concrete compression, compression of concrete mesoscopic model is established. The physical properties of the consideration of concrete material differences effect on the mechanical behavior of concrete, this paper established the concrete under uniaxial compression and unified stochastic damage constitutive relation ${ }^{[2]}$. Adjusting the two spring stiffness ratio in the mesoscopic model and friction coefficient of friction block to simulate different strength grade of concrete mesoscopic structure, the corresponding strength grade of concrete damage constitutive relations is established. In this paper, for the study of concrete under multiaxial stress state of stochastic damage constitutive relation provides a theoretical reference.

\section{Microscopic damage model}

\section{Microscopic element}

Based on double spring model, considering in the concrete aggregate and mortar material differences in physical properties, set up the concrete mesoscopic damage element model as shown in reference [3].The model consists of two springs, a friction piece and a sliding blocks, including 1 spring and friction block in the simulation of concrete cement mortar; Spring 2 in simulated concrete aggregate; Slide block for displacement control, that is, when the deformation of the spring 1 reach their limit deformation before friction block doesn't work. Concrete axial compression 
diagram and its concrete mesoscopic model ${ }^{[4]}$.

\section{Failure mode}

Mesoscopic unit of the whole stiffness are two springs in series stiffness, namely

$$
E=\frac{E_{1} E_{2}}{E_{1}+E_{2}}
$$

Assume $E_{2}=k E_{1}$, then $E=k E_{1} /(1+k), k$ is the stiffness ratio.

\section{Damage constitutive law}

\section{Damage parameter}

Based on the literature [1] on the analysis of the concrete under uniaxial tension stochastic damage, damage as defined along the compression direction is $_{\varepsilon}$

$$
\begin{aligned}
& D_{1}(\varepsilon)=\frac{1}{\mathrm{~A}} \lim _{Q \rightarrow \infty} \sum_{\mathrm{i}=1}^{Q} H\left(\varepsilon-\Delta_{\mathrm{i}}\right) A_{\mathrm{i}}=\int_{0}^{1} H\left(\varepsilon-\left(y_{1}\right)\right) d y_{1} \\
& D_{2}(\varepsilon)=\frac{1}{\mathrm{~A}} \lim _{Q \rightarrow \infty} \sum_{i=1}^{Q} H\left(\varepsilon-\Delta_{\mathrm{i}}\right) A_{\mathrm{i}}=\int_{0}^{1} H\left(\varepsilon-\left(y_{2}\right)\right) d y_{2}
\end{aligned}
$$

$\Delta\left(y_{1}\right) 、 \Delta\left(y_{2}\right)$ are the stochastic damage strain at $y_{1} 、 y_{2}, D_{1}(\varepsilon)$ and $D_{2}(\varepsilon)$ are damage parameters. For friction block unit, the damage variable can be represented as:

$$
D_{\mathrm{f}}(\varepsilon)=\frac{A_{\mathrm{f}}(\varepsilon)}{A}=\int_{0}^{1} H(\varepsilon-\Delta(z)) d z
$$

$A_{\mathrm{f}}(\varepsilon)$ is the damage section surface, $\Delta(z)$ is limiting strain of clutch block, $\Delta(y)$ is limiting strain of spring, $D_{\mathrm{f}}(\varepsilon)$ 也 is a stochastic damage parameter, $\varepsilon$ is Transverse tensile strain on average, $\varepsilon=v \varepsilon_{2}$.

\section{Constitutive law}

According to the principle of conservation of energy, the external force of concrete work, part of elasticity can be stored as material. But others were given along with the development of the injury and consume. Building energy conservation equation:

$$
\int_{0}^{\varepsilon} \sigma_{1}(\varepsilon) d x=W_{e}(\varepsilon)-W_{D}(\varepsilon)
$$

$W_{e}(\varepsilon)$ is the strain energy density of spring for strain $\varepsilon, W_{D}(\varepsilon)$ 为 is the strain energy density of spring, when the spring is broken for strain $\varepsilon$.

In the process of uniaxial compression, will have an irregular cracks in the other two directions ${ }^{[6]}$, so the influence coefficient are introduced to consider the factors $\alpha$, so the total damage dissipation is

$$
W_{D}(\varepsilon)=2 \alpha W_{D_{2}}(\varepsilon)
$$

According to the uniaxial compression of mesoscopic model analysis, damage energy density of the tensile direction $\varepsilon_{2}$

$$
W_{D_{2}}(\varepsilon)=\int_{0}^{\varepsilon} E x D_{2}(x) d x
$$

According to the different failure modes of concrete, it is divided into super-high strength high 
performance concrete and ordinary concrete and high strength concrete points three types don't energy calculation analysis.

\section{Verification}

In order to validate the presented stochastic damage constitutive relation, the ultra-high strength high performance concrete, high strength concrete and ordinary concrete under uniaxial tension, uniaxial compression test ${ }^{[7]}$. Mesoscopic damage variables in the model assumes that concrete obey the lognormal distribution, figure 1 respectively for C120, C30 strength grade and C80 concrete in uniaxial tension stress-strain test results with the theoretical value of contrast and damage variable function curve.
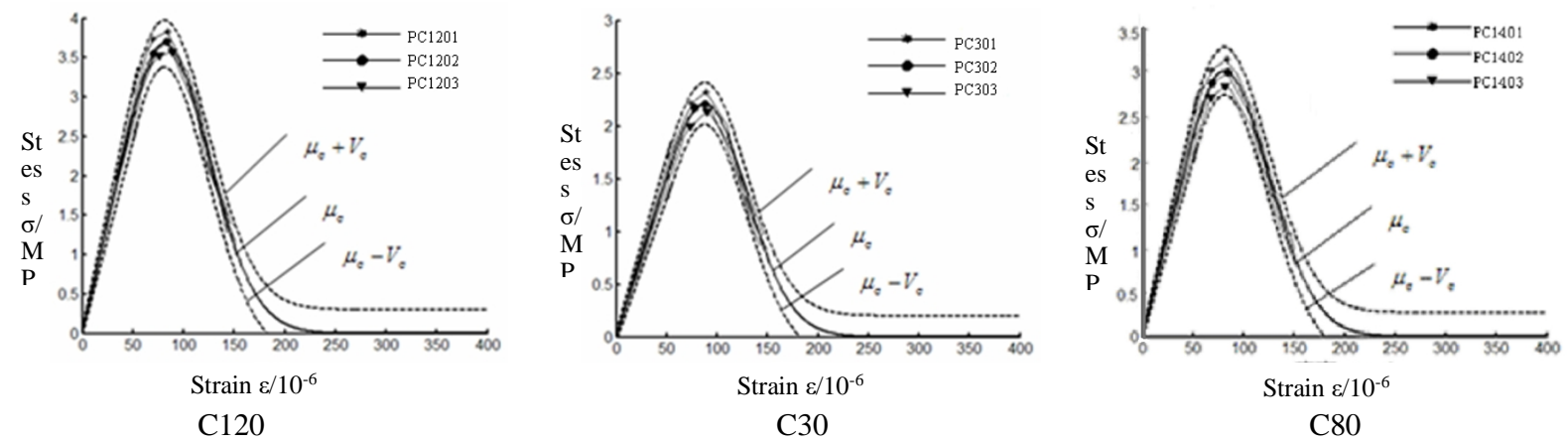

a. Stress-Strain Curve
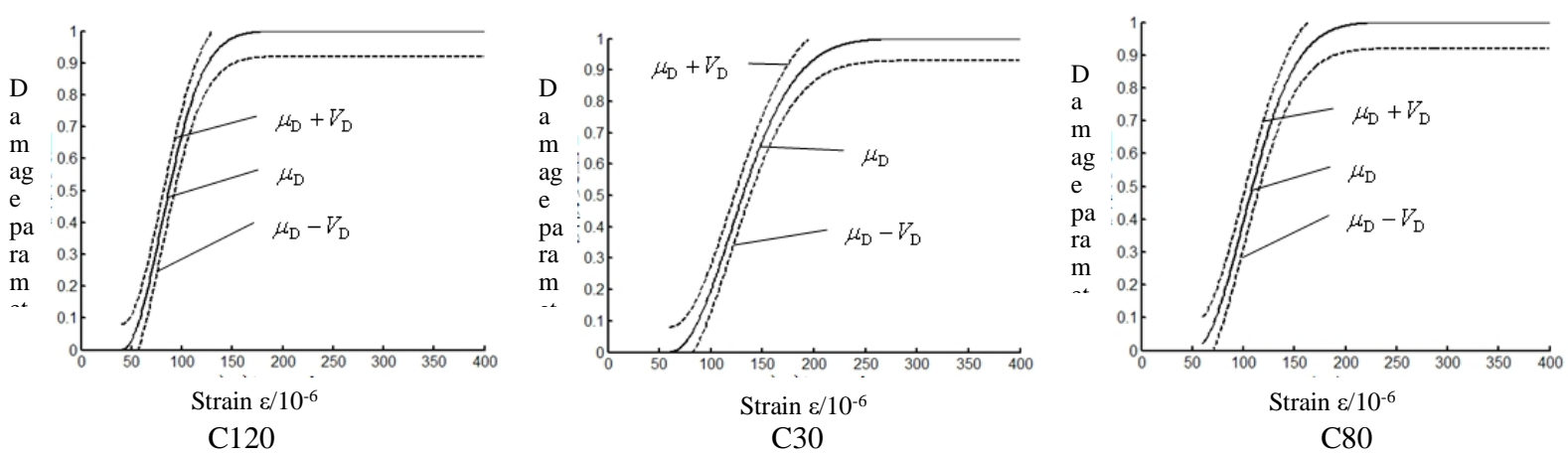

b. Damage Parameter Curve

Fig. 1 comparison between test results and theoretical results

By above knowable most of the test data in this paper to establish the mean stress - strain curve of double variance range, in the present paper suggests that the stochastic damage constitutive relation can well reflect the actual mechanical properties of concrete and its damage of randomness.

\section{Conclusion}

Mesoscopic stochastic damage model of concrete is presented in this paper, considering the differences of physical properties of concrete material, in normal concrete, high strength concrete are analyzed and the failure mechanism of super-high strength high performance concrete. Concrete unified stochastic damage constitutive relation is established. In this paper, the stochastic damage constitutive relation has the following advantages:

(1) Adjusted by spring elastic modulus relative relationship, to simulate the mesoscopic structure of different strength grade of concrete, and its damage mechanism.

(2) The proposed stochastic damage variables, considering the randomness of concrete damage, reflect the performance of concrete damage mechanics of randomness and discreteness. 
(3) Based on two spring stiffness within the micromechanical model of relative relationship, will be inside the concrete aggregate elastic modulus and the elastic modulus of mortar reduced to one of the elastic modulus of random relations.

(4) The constitutive relation is not only can reflect the average damage characteristics of concrete, but also quantitatively describe the discrete range.

\section{Reference}

[1] Liu bin. Study on Stochastic Damage Constitutive of Higher strength and high performance Concrete [D]. Xi'an: Xi'an University of Architecture and Technology. 2010.

[2] Li Jie, Lu Chaohui, Zhang Qiyun. Study on Stochastic Damage Constitutive Law for Concrete Material Subjected to Uniaxial Compressive Stress [J]. Shanghai: Journal of Tongji University. 2003.5, 31(5): 503-509.

[3] Guo Zhenhai, Shi Xudong. Reinforced Concrete Theory and Analyse[M]. Beijing: Tsinghua University Press, 1998.

[4] Guo Zhenhai. Strength and Deformation of Concrete-Experimental Basis and Constitutive Relation [M]. Beijing: Tsinghua University Press, 1997.

[5] Dong Yuli, Xie Heping, Zhao Peng. Experimental Study on Complete Damage Process of Concrete Under Compression [J]. Journal of Experimental Mechanics, 1995, 1(2):95-102.

[6] Che Shunli. Study on High Strength and High Performance Concrete Applied to Steel Reinforced Concrete Structure and Its Damage Constitutive Relationships [D]. Xi'an: Xi'an University of Architecture and Technology. 2010.

[7] Li Jie, ZhangQiyun. Study of Stochastic Damage Constitutive Relationship for Concrete Material [J]. Shanghai: Journal of Tongji University. 2003.5, 31(5): 1135-1141. 